\title{
SPATIAL BEHAVIOUR IN PLANE INCOMPRESSIBLE ELASTICITY ON A HALF-STRIP
}

\author{
BY \\ R. J. KNOPS (Dept. of Mathematics, Heriot-Watt University, Edinburgh, Scotland, UK) \\ AND \\ P. VILlagGIO (Dipartimento di Ingegneria Stutturale, Universitá di Pisa, Pisa, Italy)
}

\begin{abstract}
Growth and decay estimates are derived for an incompressible homogeneous isotropic elastic material occupying a plane semi-infinite strip in equilibrium under self-equilibrated loads on the base and zero traction along the lateral sides. The estimates depend upon a pair of differential inequalities for two cross-sectional line integrals related to different kinds of energy fluxes. A comparison with the exact solution shows that the estimates are somewhat conservative. The method, however, is applicable to non-rectangular plane regions.
\end{abstract}

1. Introduction. Incompressibility, although an idealisation, provides a satisfactory mathematical description of several physical materials including rubber and certain crystals. Yet, due to the difference between the respective equations, the solutions to the equations of incompressible elasticity cannot usually be derived from those for the classical compressible theory. Within the context of Saint-Venant's principle, this has led to recent renewed interest in incompressibility as shown, for example, by the important investigations for a semi-infinite cylinder of Maremonti and Russo [15], who discussed a perfectly incompressible elastic material, and that of Horgan and Payne [8] who considered nearly incompressible elastic materials. Both sets of conclusions, however, involve estimates only for the decay of the energy stored in partial volumes of the cylinder, and do not deal in any detail with the possibility of growth.

In this paper, we study an incompressible, homogeneous isotropic linear elastic material occupying a semi-infinite plane strip loaded on its base and having the lateral sides at zero traction. Replacement of area measures by cross-sectional line measures for the energy flux enables estimates to be derived for both growth and decay. In fact, two different line measures are required which are shown to satisfy a pair of differential inequalities whose independent variable is the distance of the cross section from the base. Integration leads to the desired estimates, which are obtained in terms of computable

Received June 3, 1998.

2000 Mathematics Subject Classification. Primary 74G50, 74G99. 
amplitudes and exponents. Similar treatments applied to the corresponding problem in steady Stokes flow along a chamnel, where the velocity and not the traction vanishes on the lateral boundary, also involve a pair of differential inequalities, which, however, are easier to solve (see, e.g., [10]-[12], [14]).

A smooth solution is assumed to exist so that, in particular, the base loading of the strip does not create any singularities at the corners. The summation convention is adopted throughout together with the comma notation for spatial partial differentiation. Greek indices assume values in the range 1, 2. Arguments of functions, especially in integrals, are omitted where there is no risk of confusion.

2. Statement of problem and basic inequalities. We consider a semi-infinite strip $\Omega$ of constant width $h(>0)$ and we select a Cartesian coordinate set of $\left(x_{1}, x_{2}\right)$ axes to have its origin located in the base and positive $x_{1}$-axis along one side. We denote the regions $\Omega(a, b)(a<b)$ and $L\left(y_{1}\right)$ respectively by

$$
\begin{aligned}
\Omega(a, b) & =\left\{x \in \Omega: a<x_{1}<b\right\}, \\
L\left(y_{1}\right) & =\left\{x \in \Omega: x_{1}=y_{1}, 0<x_{2}<h\right\},
\end{aligned}
$$

where $x$ denotes the pair $\left(x_{1}, x_{2}\right)$.

A linear isotropic homogeneous incompressible elastic material occupies $\Omega$ in a state of plane deformation and is maintained in equilibrium under zero body-force, zero traction on the lateral boundaries, and self-equilibrated loading distributed over the base. The behaviour of the displacement, strain and stress components at asymptotically large values of $x_{1}$ is not specified but is to be determined from the analysis.

The strain components, derived from the displacement $u_{\alpha}(x)$ by the equations

$$
e_{\alpha 3}=\frac{1}{2}\left(u_{\alpha .3}+u_{3 . \Omega}\right),
$$

are related to the stress components $\sigma_{\alpha, 3}$ by the expressions

$$
\sigma_{\alpha, 3}=2 \mu e_{\alpha_{3} 3}-p \delta_{\alpha, 3},
$$

where $\mu$ is the positive shear modulus, $p(x)$ is the pressure, and $\delta_{\alpha \beta}$ is the usual Kronecker delta.

The stress components themselves satisfy the equilibrium equations

$$
\sigma_{\alpha \beta . \beta}=0, \quad x \in \Omega,
$$

which, on noting (2.4), alternatively may be written as

$$
2 \mu e_{\alpha \beta . \beta}=p_{. \alpha}, \quad x \in \Omega,
$$

or as

$$
\mu u_{\alpha .33}=p_{. \alpha}, \quad x \in \Omega,
$$

on incorporating the incompressibility condition

$$
u_{\alpha . \alpha}=e_{\alpha \alpha}=0, \quad x \in \Omega .
$$

Vanishing traction on the lateral sides requires

$$
\sigma_{\alpha 2}\left(x_{1}, 0\right)=\sigma_{\alpha 2}\left(x_{1}, h\right)=0, \quad x_{1} \geq 0,
$$


while self-equilibration of the loads over the base implies the relations:

$$
\int_{L(0)} \sigma_{\alpha 1} d x_{2}=\int_{L(0)} x_{2} \sigma_{11} d x_{2}=0
$$

which with the help of (2.5) and (2.9) gives

$$
\int_{L\left(x_{1}\right)} \sigma_{\alpha 1} d x_{2}=\int_{L\left(x_{1}\right)}\left(x_{1} \sigma_{21}-x_{2} \sigma_{11}\right) d x_{2}=0, \quad x_{1} \geq 0 .
$$

In particular, from (2.4) and (2.11) we obtain

$$
\int_{L\left(x_{1}\right)} p d x_{2}=2 \mu \int_{L\left(x_{1}\right)} e_{11} d x_{2}, \quad x_{1} \geq 0 .
$$

We associate with the region $\Omega\left(x_{1}, y_{1}\right), x_{1}<y_{1}$, two energy functionals defined by

$$
\begin{aligned}
E\left(x_{1}, y_{1}\right) & =2 \mu \int_{\Omega\left(x_{1}, y_{1}\right)} e_{\alpha \beta} e_{\alpha \beta} d x, \\
E_{1}\left(x_{1}, y_{1}\right) & =2 \mu \int_{\Omega\left(x_{1} . y_{1}\right)} e_{\alpha \beta .1} e_{\alpha \beta .1} d x .
\end{aligned}
$$

Besides several standard inequalities, we need one other which for completeness is stated here.

Steklov-Korn inequality. Let $w_{\alpha}(x)$ be a smooth vector function defined on $\Omega(z, z+k)$, where $z \geq 0$ and $k$ is a positive constant, and for given $z, k$, let $w_{\alpha}(x)$ satisfy the normalization

$$
\int_{\Omega(z, z+k)} w_{\alpha} d x=\int_{\Omega(z, z+k)}\left(w_{\alpha, \beta}-w_{\beta, \alpha}\right) d x=0 .
$$

Then there exists a computable positive constant $\Gamma$ such that

$$
\int_{L(z)} w_{\alpha} w_{\alpha} d x_{2}+\int_{L(z+k)} w_{\alpha} w_{\alpha} d x_{2} \leq \frac{\Gamma}{4} \int_{\Omega(z . z+k)}\left(w_{\alpha . \beta}+w_{\beta, \alpha}\right)\left(w_{\alpha, \beta}+w_{\beta . \alpha}\right) d x .
$$

Proof. Inequality (2.16) has been established in [1] by means of the following sequence of inequalities:

$$
\begin{gathered}
\int_{L(z)} w_{\alpha} w_{\alpha} d x_{2}+\int_{L(z+k)} w_{\alpha} w_{\alpha} d x_{2} \leq K_{1} \int_{\Omega(z, z+k)} w_{\alpha, \beta} w_{\alpha, \beta} d x+K_{2} \int_{\Omega(z, z+k)} w_{\alpha} w_{\alpha} d x \\
\leq\left(K_{1}+q^{-1} K_{2}\right) \int_{\Omega(z, z+k)} w_{\alpha, \beta} w_{\alpha, \beta} d x \\
\leq\left[K\left(K_{1}+q^{-1} K_{2}\right) / 4\right] \int_{\Omega(z, z+k)}\left(w_{\alpha, \beta}+w_{\beta, \alpha}\right)\left(w_{\alpha, \beta}+w_{\beta, \alpha}\right) d x
\end{gathered}
$$

in which $K_{1}, K_{2}$ are computable positive constants, $q$ is the first nonzero eigenvalue for the free membrane problem for $\Omega(z, z+k)$, and $K$ is Korn's constant of the second kind for $\Omega(z, z+k)$ in the incompressible case. Numerical values for these constants, which all depend upon $\Omega(z, z+k)$, are available in the literature and are used in Sec. 6 . 
Alternative proofs of (2.16) are presented in [16] and [2] and impose the normalizations

$$
\int_{\partial \Sigma_{a}} w_{\alpha} d s=\int_{\partial \Sigma_{a}}\left(x_{1} w_{2}-x_{2} w_{1}\right) d s=0
$$

where $\partial \Sigma_{a}$ is the boundary of the circle of radius $a$, centre $\left(z+\frac{1}{2} k, \frac{1}{2} h\right)$, and contained in $\Omega(z, z+k)$. But different numerical values of $\Gamma$ are usually obtained.

Each method establishes the existence of the constant $\Gamma$. Inequality (2.18) may then be derived from a variational problem for which the associated eigenvalue problem is

$$
\begin{aligned}
w_{\alpha . \alpha} & =0, & & x \in \Omega(z, z+k), \\
\Gamma\left(w_{\alpha, \beta}+w_{\beta . \alpha}\right)_{, \beta} & =p_{, \alpha}, & & x \in \Omega(z, z+k), \\
\Gamma\left(w_{\alpha, \beta}+w_{\beta, \alpha}\right) n_{\beta} & =p n_{\alpha}, & & x \in \partial \Omega \backslash[L(z) \cup L(z+k)], \\
\Gamma\left(w_{\alpha, \beta}+w_{\beta, \alpha}\right) n_{\beta}-2 w_{\alpha} & =p n_{\alpha}, & & x \in L(z) \cup L(z+k),
\end{aligned}
$$

where $n$ is the unit outward normal on $\partial \Omega(z, z+k)$. The incompressibility condition (2.8) is introduced as a constraint, $p$ acts as the Lagrange multiplier, and the normalization (2.15) is assumed.

Equations (2.21)-(2.23) are formally identical to (2.8), (2.6), and (2.9) respectively. In Sec. 6 we exploit this analogy to determine a numerical lower bound for the constant $\Gamma$.

3. Line measures. The first line measure used to derive growth and decay estimates is the energy flux across the line segment $L\left(x_{1}\right)$ defined by

$$
H\left(x_{1}\right)=\int_{L\left(x_{1}\right)} \sigma_{\alpha 1} u_{\alpha} d x_{2}, \quad x_{1} \geq 0 .
$$

Successive use of (2.5), (2.9), (2.4), and (2.7), accompanied by integration by parts, enables us to deduce the identity

$$
H\left(x_{1}+k\right)-H\left(x_{1}\right)=E\left(x_{1}, x_{1}+k\right), \quad x_{1} \geq 0,
$$

where $k$ is a positive constant. Hence, we may conclude that

$$
H^{\prime}\left(x_{1}\right)=2 \mu \int_{L\left(x_{1}\right)} e_{\alpha \beta} e_{\alpha \beta} d x_{2}, \quad x_{1} \geq 0
$$

where a superposed prime denotes differentiation with respect to the argument. It follows immediately from (3.3) that

$$
H^{\prime}\left(x_{1}\right) \geq 0, \quad x_{1} \geq 0
$$

and that $H\left(x_{1}\right)$ is nondecreasing for $x_{1} \geq 0$. Let us also note that $(2.8)$ enables $H^{\prime}\left(x_{1}\right)$ to be alternatively written as

$$
H^{\prime}\left(x_{1}\right)=4 \mu \int_{L\left(x_{1}\right)}\left(e_{22}^{2}+e_{12}^{2}\right) d x_{2}, \quad x_{1} \geq 0 .
$$

The second line measure is the nonnegative function $V\left(x_{1}\right)$ defined to be

$$
V\left(x_{1}\right)=\mu \int_{L\left(x_{1}\right)} e_{\alpha 2} e_{\alpha 2} d x_{2}+\frac{1}{4 \mu} \int_{L\left(x_{1}\right)} p^{2} d x_{2}, \quad x_{1} \geq 0 .
$$


It follows from $(2.4),(2.8),(2.9)$, and an integration by parts that

$$
V^{\prime}\left(x_{1}\right)=\int_{L\left(x_{1}\right)} \sigma_{\alpha 2} u_{\alpha, 12} d x_{2}, \quad x_{1} \geq 0,
$$

which, on using (2.9) and (2.5), may be rewritten as

$$
V^{\prime}\left(x_{1}\right)=\int_{L\left(x_{1}\right)} \sigma_{\alpha 1,1} u_{\alpha, 1} d x_{2}, \quad x_{1} \geq 0 .
$$

Hence, $V^{\prime}\left(x_{1}\right)$ may be interpreted as a first-order energy flux across $L\left(x_{1}\right)$. Further differentiation of (3.8) and use of (2.5), (2.4), and (2.8) together with an integration by parts leads to the relation

$$
V^{\prime}\left(x_{1}+k\right)-V^{\prime}\left(x_{1}\right)=E_{1}\left(x_{1}, x_{1}+k\right), \quad x_{1} \geq 0,
$$

and hence

$$
V^{\prime \prime}\left(x_{1}\right)=2 \mu \int_{L\left(x_{1}\right)} e_{\alpha \beta, 1} e_{\alpha \beta, 1} d x_{2}, \quad x_{1} \geq 0
$$

so that

$$
V^{\prime \prime}\left(x_{1}\right) \geq 0, \quad x_{1} \geq 0 .
$$

Again, from (2.8) we may express $V^{\prime \prime}\left(x_{1}\right)$ alternatively as

$$
V^{\prime \prime}\left(x_{1}\right)=4 \mu \int_{L\left(x_{1}\right)}\left(e_{22,1}^{2}+e_{12,1}^{2}\right) d x_{2}, \quad x_{1} \geq 0 .
$$

4. Differential inequalities. The following propositions establish the differential inequalities satisfied by the functions $H\left(x_{1}\right)$ and $V\left(x_{1}\right)$. Their integration is discussed in Sec. 5 .

The first requires preparatory additional notation. Let $k$ be a positive constant and let $z$ be fixed. We introduce the vector function $U_{\alpha}(x)$ by

$$
U_{\alpha}(x)=u_{\alpha}(x)+a_{\alpha}+\varepsilon_{\alpha \beta} x_{\beta} b,
$$

where $\varepsilon_{\alpha \beta}$ is the two-dimensional alternating tensor, and the constants $a_{\alpha}$ and $b$ are chosen such that

$$
\int_{\Omega(z, z+k)} U_{\alpha} d x=\int_{\Omega(z, z+k)} \varepsilon_{\alpha \beta} U_{\alpha, \beta} d x=0 .
$$

We observe that $e_{\alpha \beta}$, defined by (2.3), may also be written in the form

$$
e_{\alpha \beta}=\frac{1}{2}\left(U_{\alpha, \beta}+U_{\beta, \alpha}\right), \quad x \in \Omega .
$$

On noting the self-equilibrating conditions (2.11), we may use (4.1) to express $H\left(x_{3}\right)$ in the equivalent form

$$
H\left(x_{1}\right)=\int_{L\left(x_{1}\right)} \sigma_{\alpha 1} U_{\alpha} d x_{2},
$$

which is the expression required for the first proposition. 
Proposition 4.1. The energy flux measure $H\left(x_{1}\right)$, as defined in the form (4.4), satisfies for each $x_{1} \geq 0$ the differential inequality

$$
\left|H\left(x_{1}\right)\right| \leq 2 \mu^{\frac{1}{2}}(1+\varepsilon)^{\frac{1}{2}}\left[H^{\prime}\left(x_{1}\right)+\frac{h^{2}}{4 \pi^{2} \varepsilon} V^{\prime \prime}\left(x_{1}\right)\right]^{\frac{1}{2}}\left[\int_{L\left(x_{1}\right)} U_{\alpha} U_{\alpha} d x_{2}\right]^{\frac{1}{2}},
$$

where $\varepsilon$ is an arbitrary positive constant.

Proof. We define the vector function $v_{\alpha}(x)$ by

$$
v_{\alpha}(x)=U_{\alpha}(x)-\frac{1}{h} \int_{L\left(x_{1}\right)} U_{\alpha}\left(x_{1}, \eta\right) d \eta
$$

which satisfies the relations

$$
\int_{L\left(x_{1}\right)} v_{\alpha}\left(x_{1}, \eta\right)=0, \quad \int_{L\left(x_{1}\right)} v_{\alpha} v_{\alpha} d x_{2} \leq \int_{L\left(x_{1}\right)} U_{\alpha} U_{\alpha} d x_{2} .
$$

On appealing to (2.11) and (2.4), we may use (4.6) to equivalently express $H\left(x_{1}\right)$ as

$$
H\left(x_{1}\right)=2 \mu \int_{L\left(x_{1}\right)} e_{\alpha 1} v_{\alpha} d x_{2}-\int_{L\left(x_{1}\right)} p v_{1} d x_{2} .
$$

For each fixed $x_{1}$, we introduce the differential function $w\left(x_{2}\right)$ defined by

$$
w\left(x_{2}\right)=\int_{0}^{x_{2}} v_{1}\left(x_{1}, \eta\right) d \eta
$$

so that $w(0)=w(h)=0$, and $w_{, 2}\left(x_{2}\right)=v_{1}\left(x_{1}, x_{2}\right)$. The second term on the right of (4.8) therefore becomes

$$
-\int_{L\left(x_{1}\right)} p v_{1} d x_{2}=2 \mu \int_{L\left(x_{1}\right)}\left(e_{21,1} w-e_{22} v_{1}\right) d x_{2}
$$

where we have used the stated properties of $w\left(x_{2}\right)$ together with (2.6) and an integration by parts. Insertion of (4.10) into (4.8) and recalling (2.8) leads us to

$$
H\left(x_{1}\right)=2 \mu \int_{L\left(x_{1}\right)}\left[-2 e_{22} v_{1}+e_{12} v_{2}+e_{12,1} w\right] d x_{2}
$$

An application of the Schwarz and Poincaré inequalities then yields

$$
\begin{aligned}
\left|H\left(x_{1}\right)\right| \leq 2 \mu\left[\int _ { L ( x _ { 1 } ) } \left\{4 d_{1} e_{22}^{2}\right.\right. & \left.\left.+d_{2} e_{12}^{2}+d_{3} e_{12,1}^{2}\right\} d x_{2}\right]^{\frac{1}{2}} \\
\times & {\left[\int_{L\left(x_{1}\right)}\left\{\left(d_{1}^{-1}+h^{2} \pi^{-2} d_{3}^{-1}\right) v_{1}^{2}+d_{2}^{-1} v_{2}^{2}\right\} d x_{2}\right]^{\frac{1}{2}}, }
\end{aligned}
$$

where $d_{i}(i=1,2,3)$ are positive constants selected to satisfy

$$
d_{1}=(1+\varepsilon) d_{2}=\varepsilon \pi^{2} h^{-2} d_{3},
$$

and $\varepsilon$ is an arbitrary positive constant. On substituting the values (4.13) into (4.12) and recalling the identities (3.5) and (3.12), together with (4.7) we are led to the required inequality (4.5). 
Proposition 4.2. The nonnegative function $V\left(x_{1}\right)$, defined by $(3.5)$, satisfies the differential inequality

$$
V\left(x_{1}\right) \leq H^{\prime}\left(x_{1}\right)+\frac{h^{2}}{2 \pi^{2}} V^{\prime \prime}\left(x_{1}\right), \quad x_{1} \geq 0 .
$$

Proof. It follows from (3.6) and (3.5) that

$$
V\left(x_{1}\right)=\frac{1}{4} H^{\prime}\left(x_{1}\right)+\frac{1}{4 \mu} \int_{L\left(x_{1}\right)} p^{2} d x_{2}, \quad x_{1} \geq 0 .
$$

To estimate the second term on the right of (4.15), we introduce the function $P(x)$ defined by

$$
P(x)=p(x)-\frac{1}{h} \int_{L\left(x_{1}\right)} p d x_{2}, \quad x_{1} \geq 0
$$

so that

$$
\int_{L\left(x_{1}\right)} P d x_{2}=0, \quad P_{, 2}(x)=p_{, 2}(x), \quad x_{1} \geq 0
$$

and

$$
\int_{L\left(x_{1}\right)} p^{2} d x_{2} \leq \int_{L\left(x_{1}\right)} P^{2} d x_{2}+4 \mu^{2} \int_{L\left(x_{1}\right)} e_{22}^{2} d x_{2}, \quad x_{1} \geq 0,
$$

where we have used (2.12), (2.8) and Schwarz's inequality to obtain the second term on the right. Furthermore, on setting

$$
q\left(x_{2}\right)=\int_{0}^{x_{2}} P\left(x_{1}, \eta\right) d \eta, \quad x_{1} \geq 0,
$$

for each fixed $x_{1}$, we deduce from $(4.17),(2.6)$, and an integration by parts followed by the Schwarz and Poincaré inequalities that

$$
\int_{L\left(x_{1}\right)} P^{2} d x_{2} \leq 4 \mu^{2}\left[\left(\int_{L\left(x_{1}\right)} e_{22}^{2} d x_{2}\right)^{\frac{1}{2}}+(h / \pi)\left(\int_{L\left(x_{1}\right)} e_{12,1}^{2} d x_{2}\right)^{\frac{1}{2}}\right]^{2}, \quad x_{1} \geq 0 .
$$

Insertion of (4.20) and (4.18) into (4.15) then establishes (4.14).

5. Growth and decay estimates. The required growth and decay estimates, obtained by integration of the differential inequalities satisfied by $H\left(x_{1}\right)$ and $V\left(x_{1}\right)$, are contained in the theorems of this section. Let us first note, however, that the vector function $U_{\alpha}(x)$, defined by (4.1), satisfies the normalization conditions (4.2). Hence, for the region $\Omega(z, z+k)$, where $k$ is a positive constant and $z$ is fixed, we have from (2.16) that

$$
\begin{aligned}
\int_{L(z)} U_{\alpha} U_{\alpha} d x_{2}+\int_{L(z+k)} U_{\alpha} U_{\alpha} d x_{2} & \leq \frac{\Gamma}{4} \int_{\Omega(z, z+k)}\left(U_{\alpha, \beta}+U_{\beta, \alpha}\right)\left(U_{\alpha, \beta}+U_{\beta, \alpha}\right) d x \\
& =\frac{\Gamma}{2 \mu}[H(z+k)-H(z)],
\end{aligned}
$$


where we have appealed to (4.3) and (3.2). Thus, on allowing $z$ to become arbitrary and putting $z=x_{1}$, we may conclude that

$$
\int_{L\left(x_{1}\right)} U_{\alpha} U_{\alpha} d x_{2} \leq \frac{\Gamma}{2 \mu}\left[H\left(x_{1}+k\right)-H\left(x_{1}\right)\right], \quad x_{1} \geq 0 .
$$

The entire procedure may be repeated for the region $\Omega(z-k, z)$ and, in particular, leads to

$$
\int_{L\left(x_{1}\right)} U_{\alpha} U_{\alpha} d x_{2} \leq \frac{\Gamma}{2 \mu}\left[H\left(x_{1}\right)-H\left(x_{1}-k\right)\right], \quad x_{1} \geq k .
$$

We now consider the growth and decay estimates.

Theorem 5.1 (Growth). Suppose that either (a) $H(0)>0$; or (b) $V^{\prime}(0)>0$; or (c) $H(0)>0$ and $V^{\prime}(0)>0$. Then either $H\left(x_{1}\right), V^{\prime}\left(x_{1}\right)$, or both functions possess an exponentially increasing lower bound for sufficiently large $x_{1}$.

(Explicit lower bounds are presented in the proof.)

Proof. We consider only part (c). The proof of parts (a) and (b) is similar, but may be restricted to consideration of either only (4.5) or only (4.14) respectively.

Now, by hypothesis and (3.4), (3.11), we have

$$
\begin{aligned}
H\left(x_{1}\right) & \geq 0, \quad x_{1} \geq 0, \\
V^{\prime}\left(x_{1}\right) & \geq 0, \quad x_{1} \geq 0 .
\end{aligned}
$$

We first develop inequality (4.5). From (5.4) and (5.3) it follows that

$$
H\left(x_{1}\right) \leq[2 \Gamma(1+\varepsilon)]^{\frac{1}{2}}\left[H^{\prime}\left(x_{1}\right)+\frac{h^{2}}{4 \pi^{2} \varepsilon} V^{\prime \prime}\left(x_{1}\right)\right]^{\frac{1}{2}}\left[H\left(x_{1}\right)\right]^{\frac{1}{2}}, \quad x_{1} \geq k,
$$

which, after an application of the arithmetic-geometric mean inequality, leads to

$$
0 \leq H^{\prime}\left(x_{1}\right)+\frac{h^{2}}{4 \pi^{2} \varepsilon} V^{\prime \prime}\left(x_{1}\right)-\frac{1}{2 \Gamma(1+\varepsilon)} H\left(x_{1}\right), \quad x_{1} \geq k .
$$

A linear combination of inequalities (5.7) and (4.14) produces the inequality

$$
0 \leq H^{\prime}\left(x_{1}\right)-c_{1} H\left(x_{1}\right)+c_{2}\left[V^{\prime \prime}\left(x_{1}\right)-c_{1}^{2} V\left(x_{1}\right)\right], \quad x_{1} \geq k,
$$

where the constants $c_{1}, c_{2}$ are given by

$$
c_{1}=\frac{m}{2 \Gamma(1+\varepsilon)(1+m)}, \quad c_{2}=\frac{h^{2}(m+2 \varepsilon)}{4 \pi^{2} \varepsilon(1+m)},
$$

and $m>0$ satisfies

$$
m^{2}(m+2 \varepsilon) /(1+m)^{2}=16 \pi^{2} \Gamma^{2} \varepsilon(1+\varepsilon)^{2} / h^{2} .
$$

Hence, (5.8) may be rewritten as

$$
0 \leq\left(H e^{-c_{1} x_{1}}\right)^{\prime}+c_{2}\left[\left(V e^{c_{1} x_{1}}\right)^{\prime} e^{-2 c_{1} x_{1}}\right]^{\prime}, \quad x_{1} \geq k,
$$

and thus by integration we obtain

$$
\left[H(0)+c_{2} V^{\prime}(0)+c_{2} c_{1} V(0)\right] e^{c_{1} x_{1}} \leq\left[H\left(x_{1}\right)+c_{2} V^{\prime}\left(x_{1}\right)+c_{2} c_{1} V\left(x_{1}\right)\right], \quad x_{1} \geq k .
$$


Integration of (4.14) leads to

$$
\left[H(0)+\frac{h^{2}}{2 \pi^{2}} V^{\prime}(0)-\frac{h}{\pi \sqrt{2}} V(0)\right] e^{-\left(\pi \sqrt{2} x_{1} / h\right)} \leq\left[H\left(x_{1}\right)+\frac{h^{2}}{2 \pi^{2}} V^{\prime}\left(x_{1}\right)-\frac{h}{\pi \sqrt{2}} V\left(x_{1}\right)\right]
$$

where $x_{1} \geq 0$. On elimination of $V\left(x_{1}\right)$ between (5.12) and (5.13) we obtain the required result.

In the proof of part (a), we note that (5.12) holds with $V^{\prime}\left(x_{1}\right) \leq 0$, for $x_{1} \geq 0$; otherwise, if $V^{\prime}\left(x_{1}\right)>0$ for $x_{1} \geq x_{0}>0$, say, then part (c) applies. A similar remark holds for part (b).

REMARK 5.1. Let the conditions of Theorem 5.1 hold at any point $x_{1}=x_{0} \geq 0$. Then the conclusions of the theorem continue to hold for sufficiently large $x_{1} \geq x_{0}$ and by (3.2) and (3.9) imply that either $E\left(x_{1}, \infty\right)$ or $E_{1}\left(x_{1}, \infty\right)$ or both are unbounded for $x_{1} \geq 0$.

This observation is used to provide conditions for decay estimates for $H\left(x_{1}\right), V^{\prime}\left(x_{1}\right)$, and $V\left(x_{1}\right)$.

TheOREM 5.2 (Decay). Suppose that

$$
E\left(x_{1}, \infty\right)+E_{1}\left(x_{1}, \infty\right)<\infty, \quad x_{1} \geq 0 .
$$

Then

$$
H\left(x_{1}\right) \leq 0, \quad V^{\prime}\left(x_{1}\right) \leq 0, \quad x_{1} \geq 0,
$$

(b) $\left[-H\left(x_{1}\right)-c_{2} V^{\prime}\left(x_{1}\right)+c_{1} c_{2} V\left(x_{1}\right)\right] \leq e^{-c_{1} x_{1}}\left[-H(0)-c_{2} V^{\prime}(0)+c_{1} c_{2} V(0)\right], \quad x_{1} \geq 0$,

where the positive constants $c_{1}$ and $c_{2}$ are given by (5.9) and (5.10).

Proof. Assumption (5.14) together with Remark 5.1 proves part (a).

For part (b), we introduce (5.2) into (4.5) to obtain

$$
-H\left(x_{1}\right) \leq[2 \Gamma(1+\varepsilon)]^{\frac{1}{2}}\left[H^{\prime}\left(x_{1}\right)+\frac{h^{2}}{4 \pi^{2} \varepsilon} V^{\prime \prime}\left(x_{1}\right)\right]^{\frac{1}{2}}\left[-H\left(x_{1}\right)\right]^{\frac{1}{2}}, \quad x_{1} \geq 0,
$$

and then after application of the arithmetic-geometric mean inequality we conclude that

$$
0 \leq H^{\prime}\left(x_{1}\right)+\frac{h^{2}}{4 \pi^{2} \varepsilon} V^{\prime \prime}\left(x_{1}\right)+\frac{1}{2 \Gamma(1+\varepsilon)} H\left(x_{1}\right), \quad x_{1} \geq 0 .
$$

Hence, on combining with (4.14) we have

$$
0 \leq H^{\prime}\left(x_{1}\right)+c_{1} H\left(x_{1}\right)+c_{2}\left[V^{\prime \prime}\left(x_{1}\right)-c_{1}^{2} V\left(x_{1}\right)\right], \quad x_{1} \geq 0,
$$

where $c_{1}$ and $c_{2}$ are given by (5.9). Integration of (5.19) follows upon expressing it in the form

$$
0 \geq\left(H e^{c_{1} x_{1}}\right)^{\prime}+c_{2}\left[\left(V e^{-c_{1} x_{1}}\right)^{\prime} e^{2 c_{1} x_{1}}\right]^{\prime}, \quad x_{1} \geq 0,
$$

and leads immediately to (5.16). 
REMARK 5.2. The decay estimate (5.16) not only provides corresponding estimates for each of the functions $H\left(x_{1}\right), V\left(x_{1}\right)$, and $V^{\prime}\left(x_{1}\right)$, but also leads to estimates for other quantities. For example, an estimate for the mean-square over $L\left(x_{1}\right)$ of the pressure may be derived from that for $V\left(x_{1}\right)$ on noting (3.6). Inequality (5.16) also implies that $H\left(x_{1}\right), V\left(x_{1}\right)$, and $V^{\prime}\left(x_{1}\right)$ vanish in the asymptotic limit for large values of $x_{1}$. Hence, from (3.2) it therefore follows that

$$
E\left(x_{1}, \infty\right) \leq e^{-c_{1} x_{1}}\left[E(0, \infty)+c_{2} E_{1}(0, \infty)+c_{1} c_{2} V(0)\right], \quad x_{1} \geq 0,
$$

while together with (3.9) a similar bound may be deduced for $E_{1}\left(x_{1}, \infty\right)$.

REMARK 5.3. It may be concluded from (5.21) that

$$
\lim _{x_{1} \rightarrow \infty} \int_{L\left(x_{1}\right)} e_{\alpha \beta} e_{\alpha \beta} d x_{2}=0
$$

and hence

$$
\int_{L\left(x_{1}\right)} e_{\alpha \beta} e_{\alpha \beta} d x_{2}=-2 \int_{\Omega\left(x_{1}, x\right)} e_{\alpha \beta} e_{\alpha \beta, 1} d x \leq \mu^{-1} E^{\frac{1}{2}}\left(x_{1}, \infty\right) E_{1}^{\frac{1}{2}}\left(x_{1}, \infty\right),
$$

thus yielding an estimate for the mean-square of the strain.

Finally, inequality (5.2) in conjunction with (5.21) provides a decay estimate for the mean-square over $L\left(x_{1}\right)$ of the displacement taken to within a rigid body displacement.

6. Numerical evaluation. In order to assess the numerical accuracy of the estimates derived in the previous section, we compare them with the exact solution obtained by expressing the displacement in the form

$$
u_{1}\left(x_{1}, x_{2}\right)=\psi_{.2}, \quad u_{2}\left(x_{1}, x_{2}\right)=-\psi_{.1},
$$

where $\psi\left(x_{1}, x_{2}\right)$ is a stream function. Under suitable smoothness conditions on $\psi\left(x_{1}, x_{2}\right)$, it follows from the equilibrium equations (2.7) that

$$
\mu \psi_{, 1 \alpha \alpha}=p_{.2}, \quad-\mu \psi_{, 2 \alpha \alpha}=p_{.1},
$$

and hence we conclude that $\psi$ and $p$ are respectively biharmonic and harmonic functions. We therefore assume that $\psi\left(x_{1}, x_{2}\right)$ has the form

$$
\psi\left(x_{1}, x_{2}\right)=e^{-\lambda x_{1}}\left[\left(A+\lambda B x_{2}\right) \cos \lambda x_{2}+\left(C+\lambda D x_{2}\right) \sin \lambda x_{2}\right],
$$

where the constants $A, B, C, D$ are to be chosen and the constant $\lambda$ is an eigenvalue. The general solution is obtained from (6.3) by summation over the eigenvalues and its completeness may be discussed as described, for example, in [3]. In terms of (6.3), we have

$$
\begin{gathered}
p\left(x_{1}, x_{2}\right)=2 \mu \lambda^{2} e^{-\lambda x_{1}}\left[B \cos \lambda x_{2}+D \sin \lambda x_{2}\right] \\
\sigma_{12}\left(x_{1}, x_{2}\right)=-2 \mu \lambda^{2} e^{-\lambda x_{1}}\left[\left(B+C+\lambda D x_{2}\right) \sin \lambda x_{2}+\left(A-D+\lambda B x_{2}\right) \cos \lambda x_{2}\right], \\
\sigma_{22}\left(x_{1}, x_{2}\right)=2 \mu \lambda^{2} e^{-\lambda x_{1}}\left[\left(C+\lambda D x_{2}\right) \cos \lambda x_{2}-\left(A+\lambda B x_{2}\right) \sin \lambda x_{2}\right] .
\end{gathered}
$$

The boundary conditions (2.9) are satisfied on setting

$$
\lambda h \pm \sin \lambda h=0
$$


and taking $C=0, A=D$, and

$$
D=B \tan \left(\frac{\lambda h}{2}\right) \quad \text { or } \quad B=-D \tan \left(\frac{\lambda h}{2}\right),
$$

depending upon whether the plus or minus sign is respectively taken in (6.7). With this choice of parameters, the value of the function $H\left(x_{1}\right)$, for example, is given by

$$
-H\left(x_{1}\right)=-\exp \left\{-2 \lambda x_{1}\right\} H(0)
$$

the function $V\left(x_{1}\right)$ may be similarly evaluated. The nontrivial roots of (6.7) are complex and the lowest has the value $\lambda h=4.2121+2.2508 i$ (see, e.g., [9] or [16]). Thus, the exact decay rate in (6.9) is $\exp \left\{-8.4242 x_{1} / h\right\}$ (cp. [6], p. 231).

From (5.16) the corresponding estimated decay rate $c_{1}$ is given by (5.9) for the pair of positive constants $(m, \varepsilon)$ satisfying $(5.10)$ which we note implies

$$
c_{1}^{2} c_{2}=(1+m)^{-1} \text {. }
$$

We shall endeavour to select $(m, \varepsilon)$ to maximize $c_{1}$, but let us immediately observe that (5.9) directly yields the bound

$$
c_{1}<(2 \Gamma)^{-1}
$$

In order to evaluate (6.11), we require a numerical value either for $\Gamma$ or for its lower bound. We briefly consider two methods for the determination of these quantities based respectively upon the constants in the a priori inequalities (2.18) and upon the eigenvalue problem (2.21)-(2.24). The first method leads to a comparatively poor approximation due mainly to the numerical values of the constants appearing in the $a$ priori inequalities. On the other hand, the second approach improves on the first since it relies less on $a$ priori inequalities and more on exact elements of the actual solution.

Accordingly, we first have from [4] and [7] that

$$
K_{1}=h \eta / 2, \quad K_{2}=4 h^{-1}+2 \eta^{-1}, \quad q=\pi^{2} h^{-2}, \quad K=4.5,
$$

where we have chosen $k=h, \eta$ is an arbitrary positive constant, and the conjectured value of $K$ for a square has been assumed (cp. [7], [5, Eq. (5.20)]). Hence, on setting $\eta=2 \sqrt{h} / \pi$, we obtain

$$
\Gamma=K\left(K_{1}+q^{-1} K_{2}\right)=9 h[\pi \sqrt{h}+2] / \pi^{2}>18 h / \pi^{2},
$$

which yields

$$
\Gamma^{-1}<0.548 h^{-1}
$$

Hence, by (6.11), we obtain $c_{1}<0.274 h^{-1}$, which is considerably less than the exact decay rate.

Alternative estimates for the constants $\left(K_{1}+q^{-1} K_{2}\right)$ and $K$ are known (e.g., [13], [5]) but are not discussed here. Instead, we next consider the numerical estimate for a lower bound of $\Gamma$ calculated from the eigenvalue problem (2.21)-(2.24) associated with inequality (2.16). Rather than solve exactly the eigenvalue problem and thus derive a precise value for the eigenvalue $\Gamma$, we calculate an approximate value by exploiting the analogy with the boundary value problem (2.6)-(2.11) whose exact solution is given by $(6.2),(6.3)$ subject to $(6.7)$ and $(6.8)$. 
Thus, we consider the Rayleigh quotient,

$$
\begin{aligned}
R=\frac{1}{4} \int_{\Omega\left(x_{1}, x_{2}+k\right)}\left(w_{\alpha, \beta}+w_{\beta . \alpha}\right)\left(w_{\beta, \alpha}+w_{\beta . \alpha}\right) d x & \\
/ & {\left[\int_{L\left(x_{1}\right)} w_{\alpha} w_{\alpha} d x_{2}+\int_{L\left(x_{1}+k\right)} w_{\alpha} w_{\alpha} d x_{2}\right], }
\end{aligned}
$$

for functions $w_{\alpha}$ given by

$$
w_{1}=\psi_{.2}, \quad w_{2}=-\psi_{.1},
$$

where $\psi\left(x_{1}, x_{2}\right)$ is the function (6.3) for which $\lambda$ satisfies (6.7), $C=0, A=D$, and (6.8) holds.

On substituting (6.16) into (6.15) we may conclude that

$$
\Gamma^{-1} \leq \lambda \tanh \lambda k\left[1+4\left(1-\lambda^{2} h^{2}\right)^{\frac{1}{2}}\right] /\left[3+2\left(1-\lambda^{2} h^{2}\right)+2\left(1-\lambda^{2} h^{2}\right)^{\frac{1}{2}}\right]<2 \lambda .
$$

Hence, on using (6.7), we obtain $\Gamma^{-1}<8.4242 h^{-1}$, which is an improvement on (6.14) and gives the bound $c_{1}<4.2121 h^{-1}$. Thus, this calculation shows that the estimated decay rate cannot exceed half the exact rate.

An expression for the exact maximum value of $c_{1}$ is obtained on selecting the appropriate values of $m, \varepsilon$ that satisfy (5.10). A standard calculation leads to the choice $m=\varepsilon^{-1}$ where $\varepsilon$ satisfies

$$
N \varepsilon^{3}+(2 N-1) \varepsilon^{2}+N \varepsilon-1=0,
$$

and $N=4 \pi \Gamma h^{-1}$. Then,

$$
c_{1}=\left[2 \Gamma(1+\varepsilon)^{2}\right]^{-1}
$$

with $\varepsilon$ given by

$$
\varepsilon=-\frac{(2 N-1)}{3 N}+\frac{1}{2^{\frac{1}{3}} N}\left[\left\{-G+\sqrt{\left(G^{2}+4 H^{3}\right)}\right\}^{\frac{1}{3}}+\left\{-G-\sqrt{\left(G^{2}+4 H^{3}\right)}\right\}^{\frac{1}{3}}\right],
$$

where $G=-2 N^{2}(N+1) / 3, H=-\{N(2-\sqrt{3})-1\}\{N(2+\sqrt{3})+1\} / 9$.

Other estimates for $c_{1}$ are obtained on selecting particular solutions of (5.10). For example, when $\varepsilon=\frac{1}{2}$, we have

$$
m=\omega^{2}\left[1+\sqrt{\left(1+4 \omega^{-2}\right)}\right] / 2, \quad \omega=3 \sqrt{2} \pi \Gamma h^{-1},
$$

and hence

$$
c_{1}=\frac{\omega^{2}}{6 \Gamma}\left[\sqrt{\left(1+4 \omega^{-2}\right)}-1\right],
$$

which to first order becomes

$$
c_{1}=\frac{1}{3 \Gamma}<2.808 h^{-1},
$$

on appealing to (6.17).

Hence, we conclude that the estimated decay rates are somewhat inexact when compared with the actual rate. In this respect, we remark that in the corresponding threedimensional isotropic compressible elastic problem, the use of an inequality analogous to 
(2.16) also does not estimate the decay rate to a high degree of accuracy (cp. [6], p. 246). Indeed, it appears that the use of the inequality considered in [17] likewise probably leads to an estimated decay rate that is similarly conservative.

Nevertheless, despite the inexactitude, the method described here for deriving the growth and decay estimates is not restricted to rectangular regions and is applicable to general regions $\Omega$. Hence, these estimates provide useful information on the behaviour of the solution for such regions.

\section{REFERENCES}

[1] V. L. Berdichevskii, On the proof of the Saint-Venant principle for bodies of arbitrary shape, Prikl. Mat. Mech. 38, 851-864 (1974). [Jl. Appl. Math. Mech. 38, 799-813 (1975)]

[2] J. H. Bramble and L. E. Payne, Some inequalities for vector functions and applications in elasticity, Arch. Rational Mech. Anal. 11, 16-26 (1962)

[3] R. D. Gregory, The traction boundary value problem for the elastostatic semi-infinite strip; existence of solution, and completeness of the Papkovich-Fadle eigenfunctions, J. of Elasticity 10, 295-327 (1980)

[4] C. O. Horgan, Eigenvalue estimates and the trace theorem, J. Math. Anal. Appl. 69, 231-242 (1979)

[5] C. O. Horgan, Korn's inequalities and their applications in continuum mechanics, SIAM Review 37, 491-511 (1995)

[6] C. O. Horgan and J. K. Knowles, Recent developments concerning Saint-Venant's principle, T. Y. Wu and J. W. Hutchinson, eds., Advances in Applied Mechanics 23, Academic Press, New York, 1983, pp. 179-269

[7] C. O. Horgan and L. E. Payne, On inequalities of Korn, Friedrichs and Babuska-Aziz, Arch. Rational Mech. Anal. 82, 165-179 (1983)

[8] C. O. Horgan and L. E. Payne, Saint-Venant's principle in linear isotropic elasticity for incompressible and nearly incompressible materials, J. of Elasticity 46, 43-52 (1997)

[9] G. Horvay, The end problem of rectangular strips, J. Appl. Mech. 75, 87-94 (1953)

[10] R. J. Knops, End effects in fluid flows along a pipe, Proceedings of the 7th Conference on Waves and Stability in Continuous Media, Ed. by S. Rionero and T. Ruggeri, World Scientific, 1994, pp. $224-235$

[11] R. J. Knops and C. Lupoli, End effects for plane Stokes flow along a semi-infinite strip, Z. Angew. Math. Phys. 48, 905-920 (1997)

[12] R. J. Knops and C. Lupoli, End effects for non-steady Stokes flow along a pipe (to appear)

[13] J. R. Kuttler and V. G. Sigillito, Inequalities for membrane and Stekloff eigenvalues, J. Math. Anal. Appl. 23, 148-160 (1968)

[14] C. Lupoli, Some problems of spatial behaviour in continuum mechanics, Ph.D. Thesis, Department of Mathematics, Heriot-Watt University, June 1995

[15] P. Maremonti and R. Russo, On Saint-Venant's principle and related results in incompressible linear elastostatics, Ricerche di Matematica 42, 361-375 (1993)

[16] B. S. Mittelman and A. P. Hillman, Zeros of $z+\sin z$, Math. Tables and other Aids to Computation 2, 60 (1946)

[17] N. Weck, An explicit Saint-Venant's principle in three-dimensional elasticity. Ordinary and Partial Differential Equations, Dundee (1976), Lecture Notes in Mathematics, vol. 564, Springer-Verlag, New York, 1976, pp. 518-526 